\title{
Acute appendicitis and SARS-CoV-2 in children: imaging findings at a tertiary children's hospital during the COVID-19 pandemic
}

\author{
Rida Salman ${ }^{1} \cdot$ Andrew C. Sher ${ }^{1} \cdot$ R. Paul Guillerman ${ }^{1} \cdot$ Victor J. Seghers $^{1} \cdot$ J. Ruben Rodriguez ${ }^{2}$. \\ Haleh Sangi-Haghpeykar ${ }^{3} \cdot$ Ananth V. Annapragada ${ }^{1} \cdot$ Marla B. K. Sammer $^{1}$
}

Received: 20 May 2021 / Revised: 9 September 2021 / Accepted: 5 October 2021 / Published online: 5 November 2021

(c) The Author(s), under exclusive licence to Springer-Verlag GmbH Germany, part of Springer Nature 2021

\begin{abstract}
Background Evidence suggests severe acute respiratory syndrome coronavirus 2 (SARS-CoV-2) infection may be associated with appendicitis or clinical symptoms that mimic appendicitis, but it is not clear if the findings or utility of imaging in pediatric patients with suspected appendicitis have changed since the onset of the coronavirus disease 2019 (COVID-19) pandemic.

Objective To evaluate for potential differences in SARS-CoV-2 positive and SARS-CoV-2 negative pediatric patients imaged for suspected appendicitis to determine the reliability of the existing medical imaging approach for appendicitis in a population that contains both SARS-CoV-2 positive and SARS-CoV-2 negative pediatric patients.

Materials and methods Patients imaged for suspected appendicitis Apr. 1, 2020, to Dec. 31, 2020, were identified via an electronic medical records search. Differences in ultrasound (US) diagnostic performance, use of computed tomography (CT) following US, rates of appendicitis, imaging findings of appendicitis and perforation were compared between SARS-CoV-2 positive and SARS-CoV-2 negative tested patients, using pathology and surgery as reference standards for appendicitis and perforation, respectively. Fisher exact test and Student's $t$-test were used for statistical analysis.

Results One thousand, six hundred and ninety-three patients $<18$ years old met inclusion criteria, with $46 \%(772 / 1,693)$ female, 11 imaged with only CT and 1,682 with US. Comparing SARS-CoV-2 positive and SARS-CoV-2 negative patients, no statistically significant differences in sensitivity or specificity of US ( $P=1$ and $P=1$, respectively), or in the US ( $P$-values ranging from 0.1 to 1.0) or CT imaging findings ( $P$-values ranging from 0.2 to 1.0) in appendicitis were found. Perforation rates were similar between SARS-CoV-2 positive (20/57, 35.1\% perforated) and SARS-CoV-2 negative (359/785, 45.7\% perforated) patients with appendicitis $(P=0.13)$. Use of CT following first-line US was similar, with 7/125 (5.6\%) of SARSCoV-2 positive imaged with CT after US and 127/1,557 (8.2\%) of SARS-CoV-2 negative imaged with CT after US $(P=0.39)$. Conclusion In pediatric patients with suspected appendicitis, no significant difference was found in the diagnostic performance of US, CT usage or perforation rates between SARS-CoV-2 positive and SARS-CoV-2 negative patients.
\end{abstract}

Keywords Appendicitis · Appendix · Children · Computed tomography · COVID-19 · Perforation · SARS-CoV-2 · Ultrasound

\section{Introduction}

Marla B. K. Sammer

mbsammer@texaschildrens.org

1 Edward B. Singleton Department of Radiology, Baylor College of Medicine and Texas Children's Hospital, 6701 Fannin St., Suite 470, Houston, TX 77030, USA

2 Department of Surgery, Baylor College of Medicine and Texas Children's Hospital, Houston, TX, USA

3 Department of Obstetrics and Gynecology, Baylor College of Medicine, Houston, TX, USA
Although initially associated with severe acute respiratory syndrome, the disease spectrum associated with severe acute respiratory syndrome coronavirus 2 (SARS-CoV-2) has been recognized to exhibit extrapulmonary manifestations. Extrapulmonary involvement, including of the gastrointestinal (GI), cardiovascular and neurological systems, has been widely documented in children [1-4]. Specifically, nearly $25 \%$ of children with coronavirus disease 2019 (COVID-19) have presented with GI symptoms that 
may be mild and include diarrhea, vomiting and abdominal pain, or more severe, clinically described as mimicking a surgical abdomen $[1,5]$. Furthermore, children with COVID-19 have also been described with features mimicking appendicitis, and abdominal imaging is crucial in differentiating between cases of a surgical etiology (such as appendicitis) and nonsurgical etiology in guiding further management [6]. The abdominal imaging findings in SARS-CoV-2 patients, specifically with multisystem inflammatory syndrome in children, have been reported by Blumfield et al. [7] and include ascites, hepatomegaly, echogenic kidneys, bowel wall thickening, gallbladder wall thickening, mesenteric lymphadenopathy, splenomegaly and bladder wall thickening. However, it is not clear if the findings or utility of imaging in pediatric patients with suspected appendicitis have changed since the onset of the COVID-19 pandemic.

Appendicitis has long been recognized as the most common surgical emergency in children [8]. In the setting of the COVID-19 pandemic, case reports have described the clinical course and outcome of children with acute appendicitis who also tested positive for SARS-CoV-2, with some of these cases having a complicated presentation, suggesting an association between SARS-CoV-2 and acute appendicitis [9, 10]. Given these reports, there may be changes in the imaging findings in patients who are known or suspected to be infected with SARS-CoV-2 and who present with appendicitis-like symptoms, but actually have a nonsurgical condition like terminal ileitis [6].

To address concerns about the clinical impact of SARS$\mathrm{CoV}-2$ on the diagnosis of appendicitis, this retrospective study was conducted to examine three specific questions: (1) how did the diagnostic performance of ultrasound (US) for appendicitis compare between SARS-CoV-2 positive and SARS-CoV-2 negative patients, (2) in patients with appendicitis, was there a difference in the US or computed tomography (CT) findings between SARS-CoV-2 positive and SARS-CoV-2 negative patients, and (3) were there differences in the use of CT scans following US in SARSCoV-2 positive compared to SARS-CoV-2 negative patients? To contextualize the relevance of imaging findings during this time period, the rates of perforation between SARS$\mathrm{CoV}-2$ positive and SARS-CoV-2 negative patients, as well as the rates of appendicitis between SARS-CoV-2 positive and SARS-CoV-2 negative patients and between 2020 and the previous 2 years, were compared.

\section{Materials and methods}

Our institutional review board approved this retrospective study, and patient consent was waived.

\section{Data acquisition}

The medical charts of all patients younger than 18 years who underwent US or CT for suspected appendicitis between the local onset of the COVID-19 pandemic on Apr. 1, 2020, and Dec. 31, 2020, were retrospectively reviewed. Patients were identified through an electronic medical record search of all ultrasounds ordered for appendicitis, via a unique imaging code for abdominal ultrasounds ordered for appendicitis at our institution. CTs of the abdomen and pelvis performed for the following indications provided in our structured order entry system were also identified: "Abd pain, appendicitis suspected," "Abd pain, appendicitis suspected, US equivocal" or "RLQ pain, appendicitis suspected, US equivocal." At our institution, if US is nondiagnostic or equivocal for appendicitis, it may be followed with CT, depending on the suspicion for appendicitis based on both the US findings and other clinical factors. We do not use magnetic resonance imaging as an initial or secondary diagnostic modality in children with suspected appendicitis. Patients who received imaging at outside institutions with no subsequent imaging exams at our institution were not included.

Medical records of patients imaged for appendicitis were queried to identify those who had a nasopharyngeal swab SARS-CoV-2 real-time reverse transcriptase polymerase chain reaction (rRT-PCR) test performed within 2 days before or after the imaging. Testing was performed in all patients who were surgically treated for appendicitis, per institutional policy to test all patients before general anesthesia. The criteria for testing other patients were typically at the discretion of the ordering providers. Indications for testing patients included those with fever, new onset cough or shortness of breath, loss of taste and smell, and known exposure to SARS-CoV-2. The final study population consisted of patients who were both imaged for suspected appendicitis and were tested for SARS-CoV-2.

The number of patients who had US for appendicitis compared to total emergency care visits between Apr. 1 and Dec. 31 of both 2018 and 2019 as well as the positive appendicitis rate on these US studies were tabulated and compared.

\section{Ultrasound and computed tomography reporting for appendicitis}

The following scoring system for appendicitis on US has been used at our institution since 2014, including for the reports of this study: $1=$ normal completely visualized appendix, $2=$ normal partially visualized appendix, $3=$ nonvisualized appendix, $4=$ equivocal, 
$5 \mathrm{a}=$ non-perforated appendicitis and $5 \mathrm{~b}=$ perforated appendicitis [11]. Occasionally, reports may not provide a score, or may not use the standard scoring system, at the discretion of the interpreting radiologist. For example, a patient with appendicitis may be reported as a score of 5, without specifying $5 \mathrm{a}$ or $5 \mathrm{~b}$. At our institution, equivocal results (score 4) include borderline enlargement of the appendix with no significant surrounding inflammatory changes or a normal-appearing appendix with periappendiceal fat stranding or fluid. Findings on US are reported in a standardized template at our institution, including if the appendix is fluid-filled, is compressible, contains an appendicolith, demonstrates wall hyperemia, demonstrates wall thickening, shows increased conspicuity of the periappendiceal fat or demonstrates the presence or absence of an abscess. The radiologists' reported findings for patients diagnosed with appendicitis were tabulated and compared between the two groups. The US findings were tabulated as reported by the interpreting radiologist.

The US scoring system is not used for CTs. To evaluate findings in appendicitis on CT, one author (R.S., with 2 years' experience) retrospectively reviewed CTs in patients diagnosed with appendicitis and the presence or absence of the following five findings was recorded (yes/ no): fluid-filled appendix, appendicolith, wall thickening, regional inflammation and abscess.

\section{Appendicitis positivity}

For surgically treated patients, pathology was used as the gold standard for the diagnosis of appendicitis. In those who were not surgically treated for appendicitis, a lack of follow-up operative or pathological report in the electronic medical record was used to confirm the lack of appendicitis. Follow-up in the medical record was between 0.1 and 274.5 days with a mean of 126.7 days. For those who went to surgery, appendicitis was confirmed in $100 \%$.

\section{Simple versus complex appendicitis}

At our institution, the surgeon classifies appendicitis as simple or perforated intraoperatively based on gross inspection. This data is stored in an institutional database and was retrieved.

\section{Statistical analysis}

Statistical analysis was performed using SAS (version 9.4; SAS Institute, Cary, NC), R (version 4.04; R Foundation for Statistical Computing, Vienna, Austria) and the epiR package (version 2.0.19; R Foundation for Statistical Computing). Statistical significance was defined as $P \leq 0.05$.

Patient age was the only continuous variable. It was tested for normality, specified as mean \pm standard deviation and compared using the Student's $t$-test. All rates and diagnostic performance metrics were calculated with $95 \%$ confidence intervals $(\mathrm{CI})$ and compared using the Fisher exact test.

For calculating US diagnostic performance metrics, scores of $5,5 \mathrm{a}$ and $5 \mathrm{~b}$ were considered positive, scores of 1 and 2 negative, and scores of 3 and 4 indeterminate. Sensitivity and specificity were computed using only the positive and negative scores. Additionally, rates of indeterminate scores or no score and rates of confirmed appendicitis among patients receiving indeterminate scores or no score were calculated and compared between SARS-CoV-2 positive and negative patients. Finally, rates of the distinct imaging findings on US or CT in patients with appendicitis were compared between the two groups.

\section{Results}

Three thousand, two hundred and thirty-seven patients were imaged for the suspicion of appendicitis, of whom 1,693 met the inclusion criteria of also having a SARS-CoV-2 test (Table 1). Ages ranged from 9 months to 17.9 years. Fortysix percent $(772 / 1,693)$ were female. The age and sex of SARS-CoV-2 positive and SARS-CoV-2 negative patients

Table 1 Summary of patients imaged for appendicitis

\begin{tabular}{|c|c|c|c|c|}
\hline & & \multicolumn{2}{|c|}{ Tested $(n=1,693)$} & \multirow{2}{*}{$\begin{array}{l}\text { Not } \\
\text { tested, } \\
\text { excluded }\end{array}$} \\
\hline & & $\begin{array}{l}\text { SARS-CoV-2 } \\
\text { positive }\end{array}$ & $\begin{array}{l}\text { SARS-CoV-2 } \\
\text { negative }\end{array}$ & \\
\hline \multirow[t]{3}{*}{ Imaged for suspicion of appendicitis $(n=3,237)$} & Positive appendicitis & 57 & 785 & 0 \\
\hline & $\begin{array}{l}\text { Simple } \\
\text { Perforated }\end{array}$ & $\begin{array}{l}37 \\
20\end{array}$ & $\begin{array}{l}426 \\
359\end{array}$ & \\
\hline & Negative appendicitis & 70 & 781 & 1,544 \\
\hline
\end{tabular}

More than half $(52.3 \%)$ of all patients imaged for suspected appendicitis were tested for severe acute respiratory syndrome coronavirus 2 (SARSCoV-2) 
grouped by appendicitis and perforation diagnoses are summarized in Table 2. No statistically significant difference between age and sex demographics was found between any of the groups.

Of the 1,693 included patients, 2 SARS-CoV-2 positive and 9 SARS-CoV-2 negative patients had only CT abdomen and pelvis with no previous US. These were patients between 15 years old and 17 years old with a weight range between 94 and $146 \mathrm{~kg}$, in whom US would likely be limited for evaluating the appendix. For the 1,682 patients who underwent US, scores are tabulated in Table 3 for appendicitis positive and negative patients. US diagnostic performance metrics are given in Table 4. No statistically significant difference was found in the sensitivity or specificity of US for diagnosing appendicitis between the SARSCoV-2 positive and SARS-CoV-2 negative patients. Furthermore, false-positivity rates were similar between the two groups $(1.4 \%, 1 / 69$ false positive in SARS-CoV-2 positive vs. $2.1 \%, 16 / 775$ in SARS-CoV-2 negative, $P=1$, in patients reported as appendicitis on US, but negative for appendicitis per reference standard). No significant difference was found in the rate at which radiologists used an indeterminate

Table 2 Comparison of age and sex between severe acute respiratory syndrome coronavirus 2 (SARS-CoV-2) positive and SARS-CoV-2 negative patients with and without appendicitis

\begin{tabular}{|c|c|c|c|c|c|c|}
\hline & $\begin{array}{l}\text { SARS-CoV-2 positive } \\
\text { Mean age in years (SD) }\end{array}$ & $\begin{array}{l}\text { SARS-CoV-2 negative } \\
\text { Mean age in years (SD) }\end{array}$ & $P$-value & $\begin{array}{l}\text { SARS-CoV-2 positive } \\
\text { Sex F ( } \%)\end{array}$ & $\begin{array}{l}\text { SARS-CoV-2 negative } \\
\text { Sex F }(\%)\end{array}$ & $P$-value \\
\hline No appendicitis & $11.4(4.2)$ & $10.4(4.6)$ & 0.05 & $44 / 70(62.8 \%)$ & $403 / 781(51.6 \%)$ & 0.08 \\
\hline Positive for appendicitis & $10.6(3.4)$ & $10.7(3.6)$ & 0.73 & $25 / 57(43.9 \%)$ & $300 / 785(38.2 \%)$ & 0.40 \\
\hline Simple appendicitis & $11.2(3.4)$ & $11.0(3.3)$ & 0.68 & $17 / 37(45.9 \%)$ & $175 / 426(41.1 \%)$ & 0.60 \\
\hline Perforated appendicitis & $9.4(3.3)$ & $10.5(3.9)$ & 0.16 & $8 / 20(40.0 \%)$ & $125 / 359(34.8 \%)$ & 0.64 \\
\hline
\end{tabular}

$F$ female, $S D$ standard deviation

Table 3 Ultrasound scores for appendicitis positive and negative patients broken down by severe acute respiratory syndrome coronavirus 2 (SARSCoV-2) status

\begin{tabular}{|c|c|c|c|c|c|c|c|c|}
\hline & \multicolumn{8}{|c|}{ Ultrasound scores $^{\mathrm{a}}$} \\
\hline & 1 & 2 & 3 & 4 & $5^{\mathrm{b}}$ & $5 a$ & $5 b$ & No score ${ }^{c}$ \\
\hline \multicolumn{9}{|l|}{ Positive for appendicitis } \\
\hline SARS-CoV-2 positive & $\begin{array}{l}0 \\
(0 \%)\end{array}$ & $\begin{array}{l}0 \\
(0 \%)\end{array}$ & $\begin{array}{l}3 \\
(5.4 \%)\end{array}$ & $\begin{array}{l}1 \\
(1.8 \%)\end{array}$ & $\begin{array}{l}0 \\
(0 \%)\end{array}$ & $\begin{array}{l}40 \\
(72.7 \%)\end{array}$ & $\begin{array}{l}11 \\
(20.0 \%)\end{array}$ & $\begin{array}{l}1 \\
(1.8 \%)\end{array}$ \\
\hline SARS-CoV-2 negative & $\begin{array}{l}3 \\
(0.4 \%)\end{array}$ & $\begin{array}{l}2 \\
(0.3 \%)\end{array}$ & $\begin{array}{l}38 \\
(4.9 \%)\end{array}$ & $\begin{array}{l}56 \\
(7.2 \%)\end{array}$ & $\begin{array}{l}9 \\
(1.2 \%)\end{array}$ & $\begin{array}{l}482 \\
(61.6 \%)\end{array}$ & $\begin{array}{l}178 \\
(22.7 \%)\end{array}$ & $\begin{array}{l}14 \\
(1.8 \%)\end{array}$ \\
\hline Total & 3 & 2 & 41 & 57 & 9 & 522 & 189 & 15 \\
\hline \multicolumn{9}{|l|}{ Negative for appendicitis } \\
\hline SARS-CoV-2 positive & $\begin{array}{l}35 \\
(50.7 \%)\end{array}$ & $\begin{array}{l}7 \\
(10.1 \%)\end{array}$ & $\begin{array}{l}25 \\
(36.2 \%)\end{array}$ & $\begin{array}{l}0 \\
(0 \%)\end{array}$ & $\begin{array}{l}0 \\
(0 \%)\end{array}$ & $\begin{array}{l}1 \\
(1.4 \%)\end{array}$ & $\begin{array}{l}0 \\
(0 \%)\end{array}$ & $\begin{array}{l}1 \\
(1.4 \%)\end{array}$ \\
\hline SARS-CoV-2 negative & $\begin{array}{l}408 \\
(52.6 \%)\end{array}$ & $\begin{array}{l}93 \\
(12.0 \%)\end{array}$ & $\begin{array}{l}211 \\
(27.2 \%)\end{array}$ & $\begin{array}{l}37 \\
(4.8 \%)\end{array}$ & $\begin{array}{l}0 \\
(0 \%)\end{array}$ & $\begin{array}{l}1 \\
(0.1 \%)\end{array}$ & $\begin{array}{l}15 \\
(1.9 \%)\end{array}$ & $\begin{array}{l}10 \\
(1.3 \%)\end{array}$ \\
\hline Total & 443 & 100 & 236 & 37 & 0 & 2 & 15 & 11 \\
\hline
\end{tabular}

a $1=$ normal completely visualized appendix, $2=$ normal partially visualized appendix, $3=$ nonvisualized appendix, $4=$ equivocal, $5 \mathrm{a}=$ non-perforated appendicitis, $5 \mathrm{~b}=$ perforated appendicitis

${ }^{\mathrm{b}}$ Score of 5 , not differentiated between $5 \mathrm{a}$ or $5 \mathrm{~b}$, by reporting radiologist

${ }^{\mathrm{c}}$ No score provided by reporting radiologist

Table 4 Ultrasound performance metrics for the diagnosis of appendicitis compared between severe acute respiratory syndrome coronavirus 2 (SARS-CoV-2) positive and SARS-CoV-2 negative patients

\begin{tabular}{|c|c|c|c|c|c|}
\hline & \multicolumn{2}{|c|}{ SARS-CoV-2 positive } & \multicolumn{2}{|c|}{ SARS-CoV-2 negative } & \multirow[t]{2}{*}{$P$-value } \\
\hline & $\%$ (rate) & $95 \% \mathrm{CI}$ & $\%$ (rate) & $95 \% \mathrm{CI}$ & \\
\hline Sensitivity & $100 \%(51 / 51)$ & $93.0-100 \%$ & $99.3 \%(669 / 674)$ & $98.3-99.8 \%$ & 1.0 \\
\hline Specificity & $97.7 \%(42 / 43)$ & $87.7-99.9 \%$ & $96.9 \%(501 / 517)$ & $95.0-98.2 \%$ & 1.0 \\
\hline
\end{tabular}

CI confidence interval 
score or no score between SARS-CoV-2 positive and SARSCoV-2 negative patients (Table 5). Additionally, no significant difference was found in the appendicitis positivity rate among patients with an indeterminate score or with no score between SARS-CoV-2 positive and SARS-CoV-2 negative patients (Table 5).

Ultrasound findings in SARS-CoV-2 positive and SARS$\mathrm{CoV}-2$ negative patients with pathologically proven appendicitis are detailed on Table 6. There was no statistically significant difference in the seven US findings between the two groups including a noncompressible appendix, fluidfilled appendix, appendicolith, appendiceal wall thickening, appendiceal hyperemia, increased periappendiceal fat conspicuity or abscess.

In those diagnosed with appendicitis, perforation rates were not found to be significantly different (SARS-CoV-2 positive: $20 / 57,35.1 \%$ perforated; SARS-CoV-2 negative: $359 / 785,45.7 \%$ perforated; $P=0.13)$. On US, $6 / 20(32 \%)$ SARS-CoV-2 positive perforated appendicitis patients received a score of $5 \mathrm{~b}$ (perforated on US), compared to $153 / 357$ (42.8\%) of SARS-CoV-2 negative patients $(P=0.35)$. Twenty-eight of $36(77.8 \%)$ SARS-CoV-2 positive non-perforated appendicitis patients received a score of 5a, compared to 322/425 (75.8\%) of SARS-CoV-2 negative patients $(P=1)$.

For the 1,682 patients who underwent initial US, no difference was found in the number of CTs ordered following the initial US between SARS-CoV-2 positive $(7 / 125=5.6 \%)$ and SARS-CoV-2 negative $(127 / 1,557=8.2 \%)$ patients $(P=0.39)$. In the majority of cases, CT was performed when US failed to show the appendix (score 3 ) or when CT showed equivocal findings (score 4). CT imaging findings of SARS-CoV-2 positive and SARS-CoV-2 negative patients with appendicitis are detailed in Table 7, with no significant difference in the findings in appendicitis between the two groups.

The rate of appendicitis was not found to be statistically different between patients tested for SARS-CoV-2 and found to be positive $(57 / 127=44.9 \%)$ or negative $(785 / 1,566=50.1 \% ; P=0.27)$. Finally, the rate of patients

Table 5 The rates at which the indeterminate and no scores were reported compared between severe acute respiratory syndrome coronavirus 2 (SARS-CoV-2) positive and SARS-CoV-2 negative patients, including the appendicitis rates for patients with indeterminate or no score reports

\begin{tabular}{llll}
\hline & SARS-CoV-2 positive & SARS-CoV-2 negative & $P$-value \\
\hline Rate of radiologists using an indeterminate score & $23.2 \%(29 / 125)$ & $22.0 \%(342 / 1,557)$ & 0.74 \\
Rate of radiologists using no score & $1.6 \%(2 / 125)$ & $1.5 \%(24 / 1,557)$ & 1 \\
Appendicitis positivity rate with indeterminate score & $13.8 \%(4 / 29)$ & $27.5 \%(94 / 342)$ & 0.13 \\
Appendicitis positivity rate with no score & $50 \%(1 / 2)$ & $58.3 \%(14 / 24)$ & 1 \\
\hline
\end{tabular}

Table 6 Ultrasound findings in patients with pathologically proven appendicitis who were imaged with ultrasound, compared based on severe acute respiratory syndrome coronavirus 2 (SARS-CoV-2) status

\begin{tabular}{llllllll}
\hline & Fluid-filled & Noncompressible & Appendicolith & Wall hyperemia & Wall thickening & $\begin{array}{l}\text { Increased fat } \\
\text { conspicuity }\end{array}$ & $\begin{array}{c}\text { Abscess } \\
\text { SARS-CoV-2 positive }\end{array}$ \\
& $49 / 56$ & $52 / 56$ & $21 / 56$ & $48 / 56$ & $50 / 56$ & $52 / 56$ \\
& $(87.5 \%)$ & $(92.8 \%)$ & $(37.5 \%)$ & $(85.7 \%)$ & $(89 \%)$ & $(92.8 \%)$ & $(1.8 \%)$ \\
SARS-CoV-2 negative & $650 / 782$ & $661 / 782$ & $295 / 782$ & $607 / 782$ & $650 / 782$ & $691 / 782$ & $47 / 782$ \\
& $(83.1 \%)$ & $(84.5 \%)$ & $(37.7 \%)$ & $(77.6 \%)$ & $(83.1 \%)$ & $(88.3 \%)$ & $(6.0 \%)$ \\
$P$-value & 0.46 & 0.12 & 1 & 0.18 & 0.27 & 0.39 & 0.36 \\
\hline
\end{tabular}

Numbers represent the number of patients in whom the finding was present on ultrasound/the total number in each group

Table 7 Computed tomography (CT) findings in patients with pathologically proven appendicitis who were imaged with CT, compared based on severe acute respiratory syndrome coronavirus 2 (SARSCoV-2) status

\begin{tabular}{llllll}
\hline & Fluid-filled & Appendicolith & Wall thickening & $\begin{array}{l}\text { Regional } \\
\text { inflammation }\end{array}$ & Abscess \\
\hline SARS-CoV-2 positive & $5 / 5$ & $3 / 5$ & $4 / 5$ & $5 / 5$ & $0 / 5$ \\
& $(100 \%)$ & $(50.0 \%)$ & $(80.0 \%)$ & $(100 \%)$ & $(0 \%)$ \\
SARS-CoV-2 negative & $52 / 64$ & $18 / 64$ & $42 / 64$ & $54 / 64$ & $12 / 64$ \\
& $(81.3 \%)$ & $(28.1 \%)$ & $(65.6 \%)$ & $(84.4 \%)$ & $(18.8 \%)$ \\
$P$-value & 0.58 & 0.16 & 0.66 & 1 & 0.58 \\
\hline
\end{tabular}

Numbers represent the number of patients in whom the finding was present on CT/the total number in each group 
imaged with US for suspected appendicitis compared to total emergency care visits was statistically significantly higher in 2020 compared to both 2018 and $2019(P<0.0001$ for both years). The positivity rate for appendicitis among those receiving ultrasounds also was statistically significantly higher in 2020 compared to both 2018 and $2019(P<0.0001$ for both years). No statistically significant variation for the perforation rate on US between 2020 and the other years was found (Table 8).

\section{Discussion}

At our pediatric tertiary care center, when comparing patients who were SARS-CoV-2 positive versus SARSCoV-2 negative, no statistically significant differences were found in the diagnostic sensitivity or specificity of US, the findings on US, the usage of CT following US, or the findings on CT in patients imaged for suspected appendicitis. Furthermore, we found no difference in the ability to detect perforation on US between the two groups. Additionally, there was no significant difference in the appendicitis perforation rate noted intraoperatively between the two cohorts.

Appendicitis as a result of SARS-CoV-2 could be attributed to a few potential factors. Intestinal cells host SARS-CoV-2 through the angiotensin-converting enzyme 2 (ACE2) receptors that are present in epithelial cells of the small intestine and colon, neuronal cells in the enterocytes and glandular cells of the appendix, and also through the transmembrane protease serine 2 (TMPRSS2) enzyme expressed in intestinal epithelial cells [12]. The copious co-expression of SARS-CoV-2 entry genes ACE2 and TMPRSS2 throughout the intestinal cells may explain GI infection with COVID-19 [13]. Furthermore, previous studies on pediatric appendicitis show that viruses can induce lymphoid hyperplasia in the appendix leading to obstruction and inflammatory changes of appendicitis [14].

Our similar rates of appendicitis in SARS-CoV-2 positive and SARS-CoV-2 negative patients coupled with a similar sensitivity for identifying appendicitis on US demonstrate that despite the theoretical propensity for SARS-CoV-2 to invade intestinal cells and cause appendicitis, imaging presentations on US and CT as shown in our study are similar. However, the ability to identify perforated from nonperforated appendicitis on US is challenging, as previously described [15]. Here, it also proved similarly limited in both the SARS-CoV-2 positive and SARS-CoV-2 negative cohorts; the rates of $5 \mathrm{~b}$ scores on US in SARS-CoV-2 positive and SARS-CoV-2 negative patients are $20 \%$ and $23 \%$, respectively, vs. surgically determined perforation rates of $35 \%$ and $46 \%$, respectively, which further suggests that the imaging appearance of appendicitis in SARS-CoV-2 positive patients is similar to noninfected patients.

Further discussion of the similar perforation rates found in patients with appendicitis who were SARS-CoV-2 positive and SARS-CoV-2 negative is warranted. Before the 2020 pandemic, a perforation rate reaching up to $40 \%$ in pediatric patients in the United States was reported [8]. However, in the context of the COVID-19 pandemic, without discussing the COVID-19 status of the patients, other institutions have reported an increase in complicated appendicitis cases, which was attributed to late presentation and delayed diagnosis precipitated by factors including parents' fear of seeking medical care for their children in medical facilities, inappropriate clinical evaluation through telemedicine, implementation of stay-at-home advisories and postponement of elective surgeries [16, 17]. In contrast, our findings are concordant with a recent study from Italy that also did not find a significant increase in the severity of acute appendicitis in 86 children in a geographical region highly affected by COVID-19 with $32.5 \%$ of children diagnosed with complicated appendicitis [18].

Symptom duration has been identified as a predictor of appendiceal perforation, with perforation generally occurring 36-48 h after initial onset of symptoms [19]. Investigation of symptom duration in the SARS-CoV-2 positive population could be instructive, although, based on our data, this does not seem of particular additional importance since we did not notice a statistically significant difference in the perforation rate when we compared our current results to the historical data from 2018 and 2019.

In contrast, we did find higher rates of appendicitis in imaged patients and higher use of US for suspected appendicitis during 2020 compared to 2018 and 2019,

Table 8 Comparison of the number of ultrasound exams performed for suspected appendicitis and the rate of positive appendicitis (scores 5, 5a and 5b) and perforation (score 5b) on ultrasound in 2020 compared to both 2019 and 2018

\begin{tabular}{lllll}
\hline & 2018 & 2019 & 2020 & $P$-value \\
\hline \% of ultrasounds for appendicitis/emergency care visits & $3.8 \%$ & $3.5 \%$ & $4.6 \%$ & 2018 vs. 2020: $P<0.0001$ \\
& $(3,706 / 98,769)$ & $(4,171 / 120,017)$ & $(3,224 / 69,689)$ & 2019 vs. 2020: $P<0.0001$ \\
Rate of appendicitis on ultrasound & $17.4 \%$ & $16.2 \%$ & $22.3 \%$ & 2018 vs. 2020: $P<0.0001$ \\
& $(643 / 3,706)$ & $(675 / 4,171)$ & $(720 / 3,224)$ & 2019 vs. $2020: P<0.0001$ \\
Rate of perforation on ultrasound (score 5b) & $22.6 \%$ & $25.5 \%$ & $26.3 \%$ & 2018 vs. $2020: P=0.12$ \\
& $(145 / 643)$ & $(172 / 675)$ & $(189 / 720)$ & 2019 vs. $2020: P=0.76$ \\
\hline
\end{tabular}


though, overall, there were fewer exams and fewer patient encounters in 2020. Additionally, while the rates of appendicitis in tested patients who were SARS-CoV-2 positive and SARS-CoV-2 negative were comparable in 2020, a substantial proportion of the patients without appendicitis were not tested for SARS-CoV-2. Consequently, the prevalence of appendicitis in SARS-CoV-2 infection was not defined, and the significance of these findings is uncertain. However, our study was not designed to evaluate if SARS-CoV-2 directly causes appendicitis. For this, pathological investigation of appendectomy specimens for SARS-CoV-2 would be most useful.

Finally, of note, there were no negative appendectomies during the study period. All patients who underwent surgery for appendicitis had pathologically confirmed appendicitis. This might be related to either greater collaborative team care for the diagnosis of appendicitis or reluctance for surgery in equivocal cases during the COVID-19 surge.

Our study has several limitations. As a retrospective analysis, it is subject to potential data entry and collection errors. Furthermore, this resulted in a large proportion of patients negative for appendicitis who were not tested for SARS-CoV-2. Additionally, it is uncertain how knowledge of SARS-CoV-2 status played into the ordering of imaging tests by referring providers and the interpretation by radiologists. In our typical workflow, US is often, but not always, performed before other diagnostic tests, including most blood tests and other laboratory tests, such as nasal swab for SARS-CoV-2.

Also, we did not assess the interobserver variability between radiologists in diagnosis and scoring, which could have altered findings. However, the scoring system has been validated previously at our institution [11]. Another limitation was in our reference standard for the patients who were not surgically treated for appendicitis; specifically, in some patients, there was a lack of long-term follow-up to confirm negative diagnoses, and our capture methods would not allow us to identify patients who were diagnosed as nonsurgical, but later could have re-presented to an outside institution with appendicitis. Furthermore, given the difficulty in data capture from outside institutions, we did not include patients who may have been diagnosed with acute appendicitis at outside institutions but surgically treated at our facility.

Finally, since this study was conducted over several waxing and waning waves of the pandemic, the longterm significance in the setting of endemic SARS-CoV-2 infection remains uncertain. Also, as with any single institution study, our results may not be widely generalizable. However, this study does report the largest cohort of pediatric appendicitis patients since the onset of the COVID-19 pandemic, specifically evaluating their medical imaging.

\section{Conclusion}

No differences in the diagnostic performance of US, CT usage, US findings, CT findings or perforation rates between SARS-CoV-2 positive and SARS-CoV-2 negative patients were found. While testing children presenting with GI symptoms for SARS-CoV-2 is prudent, our evidence does not suggest that SARS-CoV-2 status should factor into the interpretation of US or CT in suspected pediatric appendicitis.

Acknowledgments The Texas Children's Hospital COVID-19 Imaging Taskforce (Ananth V. Annapragada, Nilesh K. Desai, R. Paul Guillerman, Thierry A.G.M. Huisman, Prakash M. Masand, Rida Salman, Gunes Orman, Amir H. Pezekhmehr, Marla B. K. Sammer and Victor J. Seghers).

\section{Declarations}

Conflicts of interest Ananth V. Annapragada owns stock in Alzeca Biosciences Inc.

\section{References}

1. Akobeng AK, Grafton-Clarke C, Abdelgadir I et al (2020) Gastrointestinal manifestations of COVID-19 in children: a systematic review and meta-analysis. Frontline Gastroenterol 12:332-337

2. CDC COVID-19 Response Team (2020) Coronavirus disease 2019 in children - United States, February 12-April 2, 2020. MMWR Morb Mortal Wkly Rep 69:422-426

3. Rodriguez-Gonzalez M, Castellano-Martinez A, Cascales-Poyatos HM, Perez-Reviriego AA (2020) Cardiovascular impact of COVID-19 with a focus on children: a systematic review. World J Clin Cases 8:5250-5283

4. Lindan CE, Mankad K, Ram D et al (2020) Neuroimaging manifestations in children with SARS-CoV-2 infection: a multinational, multicenter collaborative study. Lancet Child Adolesc Health 5:167-177

5. Bolia R, Ranjan R, Bhat NK (2020) Recognizing the gastrointestinal manifestation of pediatric coronavirus disease 2019. Indian J Pediatr 88:101-102

6. Tullie L, Ford K, Bisharat M et al (2020) Gastrointestinal features in children with COVID-19: an observation of varied presentation in eight children. Lancet Child Adolesc Health 4:e19-e20

7. Blumfield E, Levin TL, Kurian J et al (2021) Imaging findings in multisystem inflammatory syndrome in children (MIS-C) associated with coronavirus disease (COVID-19). AJR Am J Roentgenol 216:507-517

8. Baxter KJ, Nguyen HTMH, Wulkan ML, Raval MV (2018) Association of health care utilization with rates of perforated appendicitis in children 18 years or younger. JAMA Surg 153:544-550

9. Meyer JS, Robinson G, Moonah S et al (2021) Acute appendicitis in four children with SARS-CoV-2 infection. J Pediatr Surg Case Rep 64:101734

10. Alsuwailem AB, Turkistani R, Alomari M (2020) Complicated appendicitis in a pediatric patient with COVID-19: a case report. Cureus 12:e8677

11. Fallon SC, Orth RC, Guillerman RP et al (2015) Development and validation of an ultrasound scoring system for children with suspected acute appendicitis. Pediatr Radiol 45:1945-1952 
12. Villapol S (2020) Gastrointestinal symptoms associated with COVID-19: impact on the gut microbiome. Transl Res 226:57-69

13. Lee JJ, Kopetz S, Vilar E et al (2020) Relative abundance of SARS-CoV-2 entry genes in the enterocytes of the lower gastrointestinal tract. Genes 11:645

14. Rabah R (2007) Pathology of the appendix in children: an institutional experience and review of the literature. Pediatr Radiol $37: 15-20$

15. Carpenter JL, Orth RC, Zhang W et al (2017) Diagnostic performance of US for differentiating perforated from nonperforated pediatric appendicitis: a prospective cohort study. Radiology 282:835-841

16. Orthopoulos $\mathrm{G}$, Santone E, Izzo F et al (2020) Increasing incidence of complicated appendicitis during COVID-19 pandemic. Am J Surg 221:1056-1060
17. Snapiri O, Rosenberg Danziger C, Krause I et al (2020) Delayed diagnosis of paediatric appendicitis during the COVID-19 pandemic. Acta Paediatr 109:1672-1676

18. La Pergola E, Sgrò A, Rebosio F (2020) Appendicitis in children in a large Italian COVID-19 pandemic area. Front Pediatr 8:600320

19. Howell EC, Dubina ED, Lee SL (2018) Perforation risk in pediatric appendicitis: assessment and management. Pediatric Health Med Ther 9:135-145

Publisher's note Springer Nature remains neutral with regard to jurisdictional claims in published maps and institutional affiliations. 